\title{
"Mall"-deando la ciudad: 24 años de territorialización de los malls en Costa Rica
}

\author{
Andrés Jiménez Corrales
}

Invitado nacional

Universidad de Costa Rica, Costa Rica

ajimenez2812@gmail.com

Recibido: 17 de mayo 2017

Aprobado: 4 de julio 2017

\section{Andrés Jiménez Corrales}

Licenciado en Geografía por la Universidad de Costa Rica. Estudiante en la Maestría Académica en Geografía en la Universidad de Costa Rica. Actualmente trabaja como investigador y docente en la misma universidad. Entre sus intereses de investigación se encuentran: producción del espacio, territorio y procesos de territorialización, urbanismo neoliberal y disparidades urbanas.

\begin{abstract}
Resumen:
La territorialización de los malls en Costa Rica inicia en el año 1993 con la inauguración de Multiplaza Escazú, en la Gran Área Metropolitana de Costa Rica. De este año al año 2017 se contabiliza un total de 14 malls. En este artículo se hará un recorrido sobre las principales características que tienen los malls; se caracterizará la llegada del primer mall al país y se desarrollará un análisis de la evolución espacio-temporal que han tenido estas infraestructuras en la GAM. Por último, se hace un repaso de algunos de los reclamos y respuestas ciudadanas que se han dado ante la construcción de un mall.
\end{abstract}

Palabras clave: mall; centro comercial; territorialización; urbanismo neoliberal.

\section{"Mall-ding" the city: 24 years of mall territorialization in Costa Rica}

\begin{abstract}
:
The territorialisation of malls in Costa Rica began in 1993 with the inauguration of Multiplaza Escazú in the Greater Metropolitan Area of Costa Rica. From 1993 to 2017 a total of 14 malls were built. In this article, a route across the main characteristics of malls is made; the arrival of the first mall to the country is characterized and an analysis of the space-time evolution that these infrastructures have had in the GAM is developed. Finally some of the complaints and citizen responses that have been given to the construction of a mall are reviewed.
\end{abstract}

Keywords: mall; shopping center; territorialization; neoliberal urbanism. 


\section{Introducción}

n el año 2017 se cumplen 24 años de la llegada del primer mall a Costa Rica. Específicamente esta infraestructura se localizó en la Gran Área Metropolitana (GAM) ${ }^{1}$, donde al año 2011 habitaban más del 50\% de la población nacional y donde se concentraba la mayor cantidad de viviendas del país (48.7\%) (INEC, 2011 citado por Plan GAM 2013). Entre los años 1982 y 2013 la GAM vivió un proceso de expansión territorial, periodo en el que duplicó su área construida y se consolidó como una centralidad urbana (Estado de la Nación, 2015, p. 275). La urbanización de este espacio ha ocurrido de manera continua en el tiempo potenciado por la constante inversión de capital en distintos tipos de infraestructuras, a lo que se suma un crecimiento poblacional del año 1984 al 2011 de 1.288 .082 habitantes a 2.268.248 habitantes, respectivamente (INEC, 2011 citado por Plan GAM 2013).

La GAM se ha constituido en una centralidad que reúne personas y capitales, por ende es un espacio geoestratégico para la inversión. En este sentido, son los actores empresariales quienes han tenido un papel relevante en la construcción de espacios en la ciudad, ya que en los últimos 30 años han sido las empresas inmobiliarias las que han direccionado el crecimiento urbano por medio del desarrollo de residenciales, comercios y oficinas, lo cual se articuló con políticas públicas que han tenido objetivos ajenos al ordenamiento territorial, permitiendo un accionar más libre de estos actores en los procesos de urbanización (Estado de la Nación, 2015, p. 289).

En este contexto de urbanización es que los malls entran a escena. Con el tiempo, estas infraestructuras se consolidan y se constituyen en un referente de espacios de consumo y de recreación en la ciudad, es decir, se convierten en un modelo de ciudad: sirve como un medio de comparación para catalogar lo bueno y lo malo en la ciudad. Por ejemplo, el Perfil del Consumidor del año $2015^{2}$ señaló que los centros comerciales ${ }^{3}$ tienen un $94 \%$ de visita de las personas que residen en este espacio y el sector de la población que asiste más son jóvenes entre los 18 y 24 años, activos laboralmente y con un mayor nivel socioeconómico (Fallas, 2 de julio del 2015). Esto refleja un aspecto relevante donde los malls y centros comerciales se convierten en espacios excluyentes y exclusivos.

Los malls llegaron a marcar una diferencia con los otros tipos de centros comerciales que existían a ese año; esto en cuanto a sus condiciones espaciales en organización de tiendas, estética y extensión, pero también en la oferta cultural-recreativa. Estos nuevos espacios reúnen funciones de consumo y recreación confinadas en su interior, generando una delimitación con el afuera, es decir, con el espacio público.

A raíz de lo anterior, este artículo se presenta como un texto que introduce una visión general y regional de los malls en Costa Rica, tomando como caso de estudio la situación de la GAM. El artículo inicia haciendo un recorrido sobre las principales características que tienen los malls; esto permitirá crear un marco de entendimiento que contribuya al posterior desarrollo del argumento. Luego, se caracteriza la llegada del primer mall al país, desde donde se parte para denotar la evolución espacio-temporal que han tenido

$1 \quad$ Es un espacio localizado en la zona central del país, constituido como una centralidad funcional. En dicho espacio se ubica la capital del país, San José. Se compone por 31 municipios, los cuales son la unidad político-administrativa del territorio a escala local.

2 El Perfil del Consumidor es un estudio que realiza Unimer, de manera anual, para el periódico El Financiero. Al año 2016 este estudio se ha realizado en cinco ocasiones. El Financiero promulga que el estudio contempla la actitud de consumo de los costarricenses, basado en nueve categorías (Fallas, 25/9/2016).

3 Dentro de dicho porcentaje se consideran distintas tipologías de centros comerciales como son malls, plazas comerciales y centros comerciales. No es posible obtener un dato preciso para los malls.

$4 \quad$ Fue inaugurado en el año de 1956. estas infraestructuras en la GAM. A continuación, se hace un repaso de algunos de los reclamos y respuestas ciudadanas que se han dado ante la construcción de un mall y se concluye con una reflexión alrededor de la categoría de análisis de urbanismo neoliberal.

\section{Caracterizando a los malls: comprender un espacio para entender su funcionalidad}

Los malls son espacios que se caracterizan en su forma y en su contenido. La primera infraestructura de este tipo se construyó en Estados Unidos específicamente en el estado de Minneapolis. Este mall se llamó Southdale Center ${ }^{4}$ y fue concebido como una centralidad que articuló el proceso de suburbanización que se encontraba en desarrollo, el cual se intensificó a partir de la década de 1950. Estos espacio se ubicaron entre los centros de ciudad y los suburbios, característica que fue replicada en otros malls 
de la época (Salcedo y De Simone, 2013; Pérez, 2010; Finol, 2005; Amaya, 2009). El mall se constituyó en una reinvención de una centralidad que fue adaptada al proceso de urbanización predominante en esos tiempos. La creación de este espacio permitió capturar las necesidades que tenía la población suburbana de una mejor accesibilidad de bienes y servicios, por lo que asumió una serie de funciones sociales, económicas, culturales y cívicas de la ciudad.

Con el tiempo los malls se exportaron a otras latitudes, edificándose en distintas ciudades del mundo, entre ellas las Latinoamericanas. Estas formas urbanas se introdujeron en un proceso de urbanización particular en cada ciudad, lo que propició que su evolución se diera de manera diferenciada y que asumiera características propias de los lugares donde se instalaba, por ejemplo, la elección de sus nombres, los elementos decorativos y la forma de promocionarse (Pérez, 2010). Son espacios diseñados como formas urbanas poli-funcionales, creadas para fungir como centros de servicios especializados y lugares de creación de estatus social (Amaya, 2009). La gestión del espacio del mall se caracteriza por pertenecer a un solo dueño, lo que permite potenciar externalidades y hacer más rentable el espacio. Otro aspecto que se destaca es la eficiencia en la gestión del espacio de los malls, la cual se contrapone con labores que realizan las municipalidades, señalando que el mall en algunas ocasiones llega a sustituir a los gobiernos locales (Galetovic, Poduje y Sanhueza, 2009). Estos espacios comerciales se convirtieron en modelos territoriales genéricos basados en sistemas de mercantilización de la ciudad (De Simone, 2014) que potencian una interacción social basada en el consumo.

Para el caso de Costa Rica se llevó a cabo una observación participante en varios malls con lo que se obtuvo una serie de características centrales que permitan entender esta forma urbana. Como primer punto tenemos que son espacios propiedad de actores empresariales que construyen las edificaciones con capital económico transnacional o nacional; se caracterizan por encerrar la mayoría de la actividad bajo techo, lo cual representa una de las características tradicionales de los malls surgidos en Estados Unidos. Aún así, en los últimos años esto se ha transformado combinando zonas bajo techo y al aire libre, con lo que intentan promocionar la creación de una vida urbana. A su alrededor, o en su interior, se dedica un campo para el parqueo de automóviles, el cual representa uno de los medios de transporte privilegiados para asistir a estos espacios, entre otras cosas por la distancia entre las residencias de los consumidores y los malls. Además, son espacios que se encuentran debidamente delimitados con el afuera (el espacio público) por medio de infraestructura, lo que genera un tipo de frontera material y simbólica que es potenciada por la estética interna del mall. Son espacios comerciales que cuentan con servicios de seguridad privada a través de lo cual controlan los actos que se desarrollan en el adentro acorde a reglas prestablecidas.

Entre sus características básicas es que son edificaciones que ofrecen una serie de bienes y servicios por medio de tiendas comerciales, por lo que genera una relación de compra y de venta con las personas que asisten a estos espacios. Cuentan con una zona de comidas rápidas y de entretenimiento y ofrecen una variada oferta cultural, lo que representa un elemento central para convocar a gentes.

Los malls delimitan un territorio dentro del cual ejercen un control de situaciones, por ejemplo, prácticas y actividades sociales, horarios para estar, acomodos espaciales de objetos y personas, cantidad de iluminación y ventilación, nivel de temperatura del ambiente, la limpieza del espacio, las actividades culturales, el tipo de tiendas comerciales o las personas que pueden o no estar en su espacio. Por tanto, son espacios que ejercen una territorialización específica en la ciudad, la cual se entiende como el proceso que van concretando actores para ejercer un control sobre un espacio. Para ello crean una estrategia espacial mediante la cual se obtiene, se influye o se controla recursos y personas (Sack citado por Haesbaert, 2011, p. 74), que para el caso de estudio, se utiliza como medio de materialización de las actividades que realizan, que crean un imaginario del mall como prototipo de ciudad. En este sentido, los malls han sido interpretados como "nuevos" espacios públicos, que según Salcedo (2003) pueden ser considerados como espacios post-públicos ya que congregan la actividad social en espacios privados. 
En resumen los malls representan un tipo de centralidad donde confluyen personas y capitales. Debido a lo anterior, y ante un deterioro sistemático del espacio público, estos son espacios que se han convertido en lugares de encuentro y reunión de personas. Los malls han llegado a producir una geografía condicionada por intereses comerciales de lucro que tiene un reflejo en su composición espacial y las actividades que desarrolla. Para este artículo se considera a los malls como una tipología de centros comerciales, por lo que se va a utilizar como punto definitorio que su infraestructura tenga una extensión territorial igual o mayor a 10.000 metros cuadrados, además de las características señaladas.

\section{La aparición de un nuevo territorio}

La aparición de los malls en América Latina representa una ruptura al modelo de ciudad que se venía implementando antes de la década de 1980, donde era el Estado un actor principal en la construcción. Este cambio se asocia con la entrada de un urbanismo neoliberal caracterizado por promover una urbanización desde actores privados, incentivar la actividad social en espacios cerrados y desarrollar un modelo de ciudad compuesto por islas urbanas, donde se privilegia los intereses de lucro y se abandona un mantenimiento de los espacios públicos (Janoschka, 2002). Además, los procesos de neoliberalización han generado la creación de una brecha mayor entre clases sociales lo cual se traduce, a nivel espacial, en un aumento de barrios urbanos excluidos, incremento de deudores habitacionales o una profundización o sostenimiento de patrones de segregación espacial (Renna, 2010). Lo anterior ha dado como resultado un espacio urbano fragmentado donde los distintos actores hegemónicos y subalternos compiten y disputan espacios; dentro de este contexto se potencia una complicidad entre Estado y actores empresariales que en algunos casos tiene una correlación en la creación de normativa; por ejemplo en planes o directrices de planificación urbana. En fin, tal y como lo señala Renna (2010), estos procesos de neoliberalización del espacio urbano representa una causa y consecuencia que producen nuevas miserias que potencia la construcción de una ciudad polarizada.

Es a partir de mediados de la década de 1980, para el caso de Costa Rica, cuando inicia la consolidación de un nuevo estilo de desarrollo (Rovira, 2004). Este cambio tuvo como eje estructurador la firma y la entrada en vigencia de Programas de Ajuste Estructural (PAE's) que se establecieron a partir de los años 1980 (Hidalgo, 2003). Dichos instrumentos fueron utilizados para crear las condiciones institucionales para la introducción de políticas neoliberales en Costa Rica. Estas políticas impulsaron la atracción de Inversión Extranjera Directa (Hidalgo, 2003) considerada como un medio para el desarrollo. Esto fue potenciado por los deficientes o nulos instrumentos de planificación que permitieron construir vivienda y centros comerciales sin mayores restricciones en distintos puntos de la GAM. El Vigesimoprimer Informe del Estado de la Nación (2015) señala que el desarrollo residencial, comercial y de oficinas se concretaron en zonas periféricas como resultado de acciones de actores empresariales junto a políticas públicas ideadas con objetivos ajenos a la planificación territorial ( $p$. 289). Lo planteado demuestra la complicidad de actores para el desarrollo del espacio urbano y es un indicio del establecimiento de un urbanismo neoliberal en el país.

Este escenario condujo a un acelerado proceso de crecimiento periférico urbano a partir de la década de 1990. Este hecho pudo ser potenciado por tres factores: 1. crecimiento del parque automotor (pasó de 230.304 unidades en el año 1995 a 863.401 unidades en el año 2014), lo que permitía a las personas desplazarse con mayor facilidad; 2. desarrollo creciente de urbanizaciones horizontales, centros comerciales y oficentros fuera de los cantones centrales de San José, Alajuela, Cartago y Heredia; y 3. abandono de los centros de los cantones señalados (Estado de la Nación, 2015, pp. 289-291). Es decir, se crearon espacios privados como alternativa al deterioro de las condiciones físicas y estéticas de algunas ciudades. Además existió un aumento e importancia social en el uso de transporte individualizado en la ciudad y una relocalización de la inversión de capital que se trasladó de los centros de ciudades a las periferias.

El sector privado se convirtió en un actor predominante que determina, en parte, las funciones y las formas territoriales que se han venido estableciendo en la ciudad 
costarricense luego de la década de 1980. Por ejemplo, para estos años hubo un cambio en la relación entre el sector privado y el sector público en construcción de vivienda, pasando de una relación 1 a 1 en 1980, a una relación 8 a 1 en 2013 (Estado de la Nación, 2015, p. 275). Esto señala que el desarrollo de la ciudad ha estado determinado en los últimos años por el sector privado, quien tiene un claro interés en producir condiciones estructurales para generar ganancias económicas. Lo anterior se ha logrado gracias a una deficiente acción pública en el ejercicio de la planificación urbana, permitiendo que el mercado inmobiliario opere con pocas restricciones (Estado de la Nación, 2015, p. 277).

En este proceso predominante de producción de la ciudad contemporánea existe una disputa por el espacio entre empresas para la localización de sus desarrollos, lo cual es potenciado por la búsqueda de una mayor rentabilidad económica ya que, en parte, una buena ubicación representa uno de los elementos primordiales para lograr un éxito comercial (Alvarado, 2014, p. 23). Lo anterior hace que distintos desarrollos produzcan límites territoriales de influencia, ejerciendo un poder para segmentar la actividad social en ciertos espacios en la ciudad.

Tal es el caso del Grupo Roble, que es un conglomerado empresarial salvadoreño que ha ejercido acciones territoriales para ubicarse en lugares estratégicos y generar formas urbanas rentables mediante las cuales buscan obtener ganancias a través de sus desarrollos. A inicios de la década de 1990 esta empresa decide establecerse en el país, inaugurando en el año 1993 el primer mall de Costa Rica llamado Multiplaza y localizado en el cantón Escazú (figura 1). Este actor cuenta con inversiones en países de América Central y Colombia. Su territorialización la ejerce a partir de la construcción de centros comerciales, hoteles y residenciales. En este sentido, Grupo Roble destaca que sus desarrollos han propiciado un cambio en la fisonomía urbana de estos países (Grupo Roble, Sin fecha) creando territorios que establecen un consumo de espacios, objetos o servicios.

Esta empresa produce formas urbanas cerradas que tienden a la absorción de la actividad social que llega a condicionar por medio de reglas pre-establecidas. Estos espacios crean límites dentro de los cuales se ejerce un control de las relaciones y tienden a crear un modelo de ciudad. Con todo ello se produce una diferenciación espacial mediada por la tenencia de capital para el acceso y uso de las actividades de los lugares.

La construcción de Multiplaza se realiza en pleno proceso de restructuración económica y se localizada al margen de una carretera de categoría nacional: la Ruta Nacional $n^{\circ}$ 27, que con los años se constituyó en un eje vial con un elevado flujo vehicular diario (Madrigal, 17 de agosto del $2015^{6}$ ). Esta ruta vial, desde principios de la década de 1990, se perfilaba como un eje que iba a concentrar una elevada inversión de capital, pronosticando una futura reestructuración de la dinámica y el proceso de urbanización de la zona oeste de la ciudad San José (Lungo, Pérez y Piedra, 1992, pp. 66 y 67).

$5 \quad$ Este mall luego va a ser denominado Multiplaza Escazú, como una forma de diferenciarse del otro mall que lleva un nombre homónimo (Multiplaza Curridabat) y que pertenece a la misma empresa. Multiplaza Escazú va a experimentar varias ampliaciones de su infraestructura, lo que lo convertirá en uno de los malls de mayor extensión en Costa Rica, solo superado por el City Mall, inaugurado en el año 2015.

$6 \quad$ En esta nota periodística se señala que solo en el mes de junio 2015, el dinero recaudado en el peaje de Escazú, localizado a menos de un kilómetro de distancia de Multiplaza Escazú, fue de dos millones de dólares (Madrigal, 2015, Agosto

17). Entonces, para obtener un aproximado de la cantidad de vehículos que transitan por este peaje, tan solo en un mes, se procedió a dividir el monto recaudado entre el precio que paga un automóvil de dos ejes a mayo 2017 (350 colones). Esto dio como resultado más de tres millones de automóviles.
Elisa Rojas, gerente de mercadeo de Grupo Roble (citada por Briceño, 2014), señala el clima de inversión y el desarrollo social y económico del país como razón para la escogencia de su emplazamiento. Lo anterior puede ser interpretado como ventajas comparativas que tiene el país en relación con otros, lo que favorece la concreción de la inversión de capital.

Otra de las características que identifica el espacio donde se ubicó este mall, es que a inicios de la década de 1990 el distrito San Rafael del cantón Escazú era una periferia de la ciudad San José (figura 1). Los alrededores de Multiplaza tenían un desarrollo de baja densidad, con predominancia de espacios de cultivos y contaba con poca presencia de infraestructura (figura 1).

Lo anterior es indicativo para pensar que el mall se pudo convertir en un núcleo que articuló el desarrollo urbano de este espacio, convirtiéndose con el tiempo en una zona de concentración comercial. Dicha periferia se constituyó en una centralidad, materializada por una alta oferta de espacios que brindan bienes y servicios, pero también por la construcción de residenciales y condominios luego de la inauguración del mall. Esta elevada inversión de capital privado llevó a una saturación comercial, por lo que en el año 2002, menos de 10 años después de haberse inaugurado Multiplaza, 


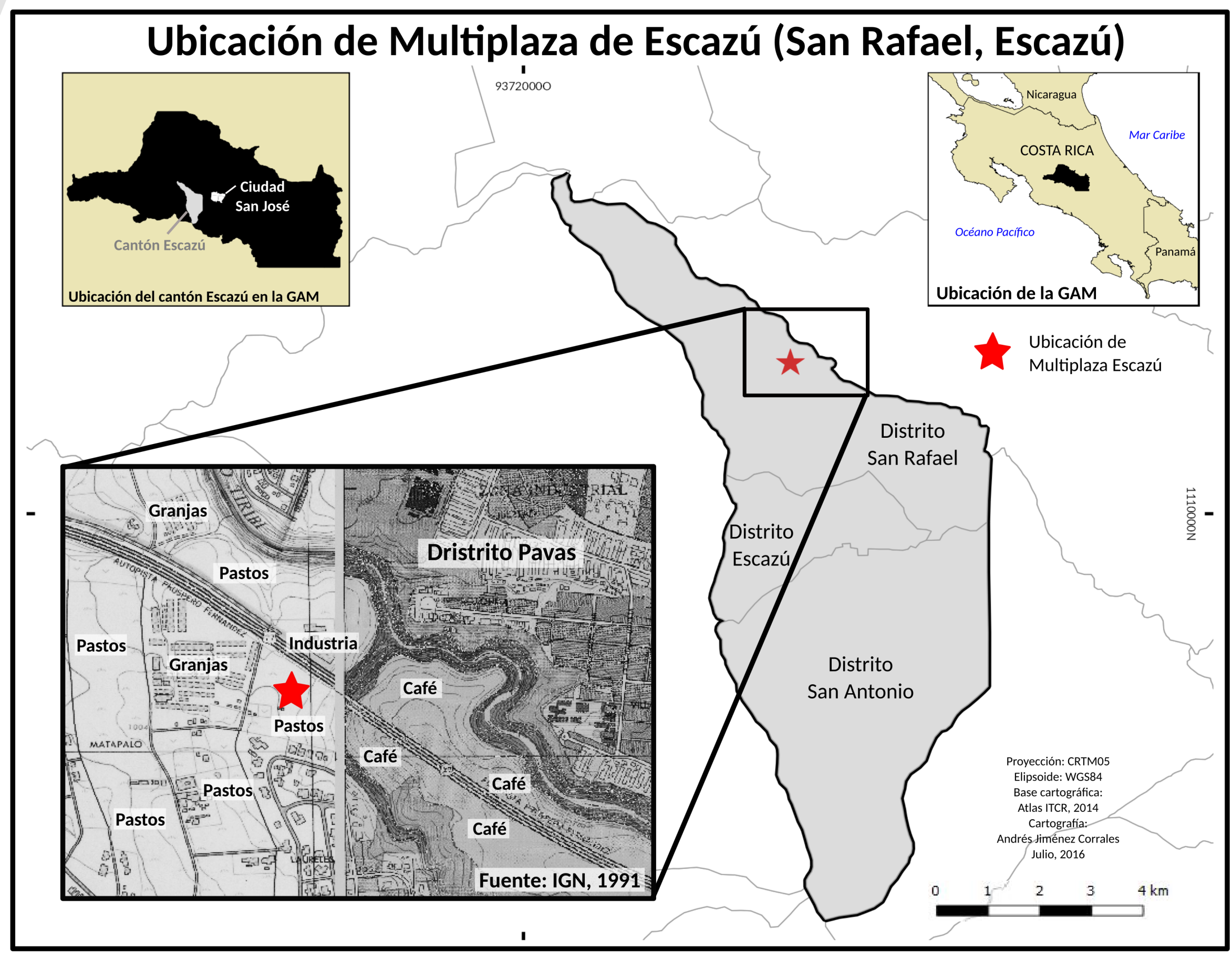

Figura 1. Ubicación del mall Multiplaza Escazú en su contexto cantonal. Se observa en el recuadro los usos de la tierra presente en los alrededores donde se va a construir el mall para el año 1991. Destaca la predominancia de usos agrícolas. Elaboración propia.
$7 \quad$ Son espacios de una extensión territorial menor a 10.000 metros cuadrados, las tiendas se encuentran alrededor de un parqueo y no ofrecen un espacio extenso de comidas ni salas de cine. Las tiendas que se localizan en estos centros comerciales no son especializadas.

$8 \quad$ En distintas conversaciones sostenidas en el marco de la investigación, tanto con académicos como público en general, destaca el hecho de considerar al mall San Pedro como el primer mall en Costa Rica. Esto llevó a reflexionar sobre las razones que crearon este imaginario, una de las cuales es porque este espacio fue primer centro comercial que se autodenomina mall. se afirmaba que en este distrito se experimentaba una sobre oferta comercial. Para ello se ponía como prueba la existencia de varios locales vacíos, con rótulos de "se alquila" o "se vende" (Brenes, 19 de abril del 2004). A pesar de dicha afirmación, la realidad señala que este espacio aún sigue siendo un punto de inversión, ya que en los últimos siete años se han construido grandes centros comerciales como Avenida Escazú y Distrito Cuatro, así como la ampliación del mall en cuestión (Fallas, 7 de septiembre del 2014; y Arce, 24 de octubre del 2009).

Multiplaza llegó a impactar en el desenvolvimiento del cantón Escazú, por lo que se afirma que "...lo que vino aquí a cambiar totalmente el asunto fue Multiplaza (...) Ahí lo que había eran unos gallineros, era totalmente un área rural" (encargado de la Oficina de Desarrollo Urbano de la Municipalidad de Escazú citado por Alvarado y Jiménez, 2014, p. 94). Grupo Roble introdujo al país un espacio que se convertiría en un nicho de mercado que, para ese entonces, era dominado por otro tipo de comercios, los cuales tenían características distintas a las ofrecidas por los malls. Dentro de ellos se encuentran los llamados centros comerciales, como es el caso del Centro Comercial Guadalupe, el cual fue el primero en su tipo ${ }^{7}$. Este centro fue inaugurado en el año 1973 (Centro Comercial Guadalupe, Sin fecha) y según Avendaño (1990) fue un espacio que ejerció un cambio en las funciones y formas precedentes, por lo que marca una ruptura en la construcción de geografías, tal como llegó a representar en su época el mall.

\section{La proliferación de un espacio de consumo}

En el año 1993, mismo año en el que se inauguró Multiplaza de Escazú, inició la construcción del mall San Pedro, en el cantón Montes de Oca. Esta infraestructura representó el segundo centro comercial de este tipo en el país, territorializándose en un espacio cercano a la ciudad de San José. Este punto permitió una mayor accesibilidad a su territorio, que además surge como una alternativa de comercio cercano a la ciudad, lo que explica por qué se piensa, en muchos casos, al mall San Pedro como el primer 
$9 \quad$ En esta contabilidad quedó por fuera los centros comerciales: American Outlet y mall Paraíso. Esto debido a que se determinó como forma de clasificación a los malls que su área de construcción sea igual o mayor a los 10.000 metros cuadrados, donde los dos casos señalados no cumplen con este requisito. Se destaca, además, que existen otras formas comerciales como las plazas comerciales, las cuales han tenido en los últimos años un auge en su construcción (Fallas, 6/2/2015). Otra forma comercial presente en la GAM son las llamadas mini-ciudades, las cuales congregan residencias, oficinas y comercio, tal es el caso de Avenida Escazú y Distrito Cuatro, ambos ubicados en el cantón Escazú. Estas formas urbanas no son parte del objeto de la investigación. mall que se construyó en Costa Rica8.

Donald Formal, dueño de este mall, quiso incursionar en el área comercial replicando una infraestructura que observó en sus viajes de negocio (especialmente en la ciudad de Miami) y considerando que era un espacio que le permitiría generar una rentabilidad económica (Corrales, 1 de mayo del 2016). Importar la idea del mall desde otras latitudes no fue problema, ya que este espacio tiene una alta capacidad de adaptación a las realidades urbanas locales (Salcedo y De Simone, 2012, p. 34).

Con la aparición de Multiplaza Escazú inicia un proceso de territorialización que instaura un tipo de función y forma urbana en el país. Lo anterior abre las posibilidades para la gestación de un tipo de control territorial que crea un acomodo del espacio-tiempo en la ciudad. De esta manera, al año 2016 se contabilizan un total de 14 malls ${ }^{9}$ en distintos cantones de la GAM (figura 2).

La aparición de estos malls no solo se dio en distintos espacios sino también en tiempos. Por esto, en la década de 1990 se contabiliza la apertura de cuatro malls; en la década de los 2000 se inauguraron, de igual manera, cuatro malls; y entre los años 2010 y 2016, entran en funcionamiento seis malls (figura 3). Este dato muestra el impulso que ha tenido en tiempos recientes el establecimiento de este tipo de espacios, ya que en los últimos seis años se han inaugurado casi la mitad de malls contabilizados para todo el periodo de estudio (1993-2016). Lo anterior permite pensar el papel que tienen estos espacios en la vida social y cultural urbana del país, concentrando personas y construyendo una imagen de ciudad.

\section{Malls en la Gran Área Metropolitana de Costa Rica (GAM), 2016}

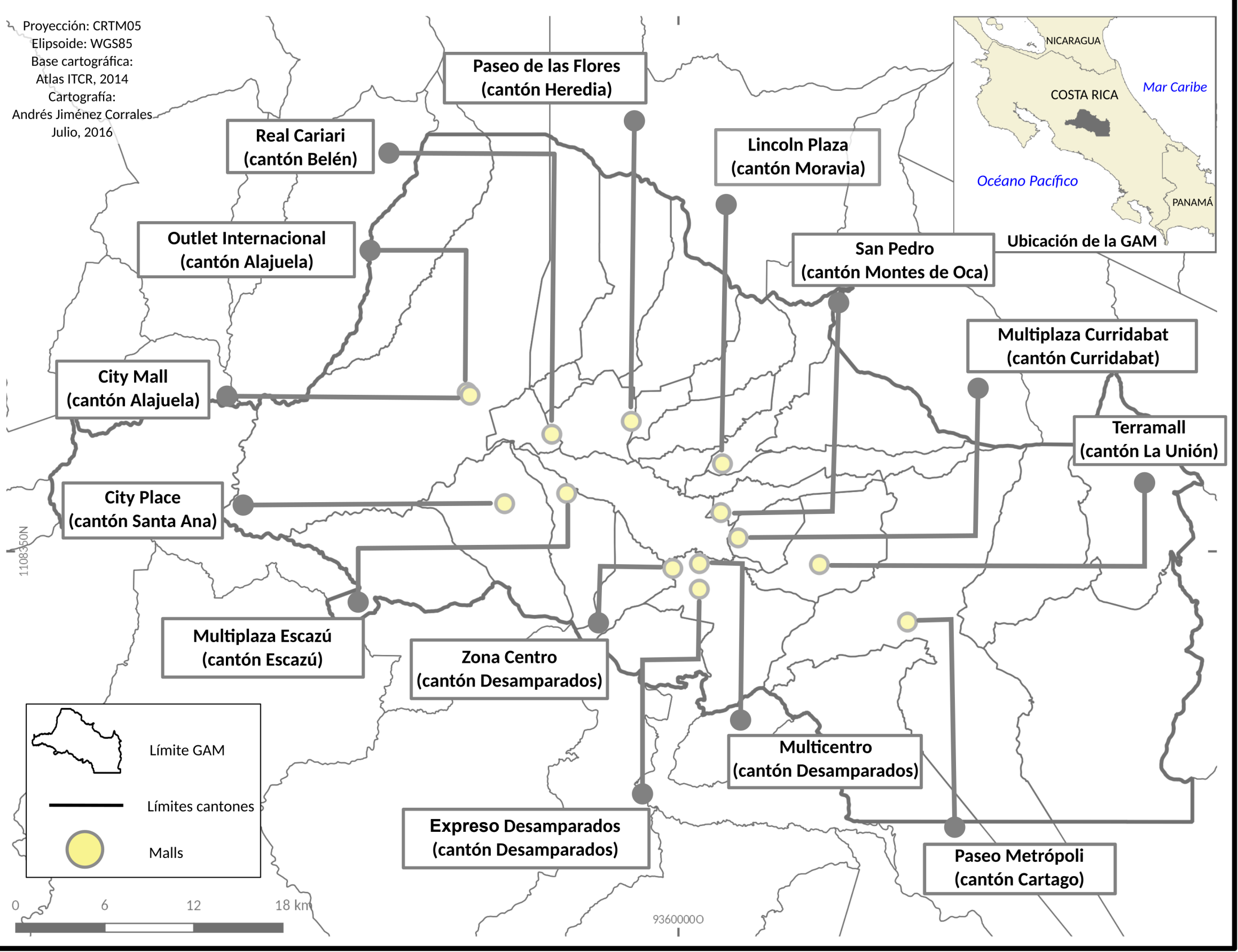


10 Esta localización es estratégica ya que presenta una buena accesibilidad, esto se refleja en que es uno de los malls que cuenta con mayor asistencia de personas al año (Corrales, 1/4/2016)

Figura 3. Ubicación de los malls según año de aparición. Elaboración propia.
Estos malls muestran una variación espacial en cuanto a los cantones donde se ubican según su década de aparición, a saber:

1. En la década de 1990: Escazú (Multiplaza de Escazú), Montes de Oca (Mall San Pedro), Belén (Plaza Real Cariari) y Alajuela (Mall Internacional).

2. En la década de 2000: Curridabat (Multiplaza del Este), La Unión (Terramall), Heredia (Paseo de las Flores) y Desamparados (Multicentro).

3. Entre los años 2010 y 2016: Cartago (Paseo Metrópoli), Moravia (Lincoln Plaza), Alajuela (City Mall), Desamparados (Zona Centro y Expreso Desamparados) y Santa Ana (City Place).

De esta información destaca el hecho de que las primeras apariciones de los malls, en la década de 1990, se realizaron principalmente en el sector oeste de la ciudad San José con excepción del mall San Pedro, localizado al este pero muy cerca de esta ciudad ${ }^{10}$. Durante la década de los años 2000, fue más bien el sector este de la ciudad el que tuvo una predominancia, presentando solo un caso en el sector noroeste (mall Paseo de las Flores). Mientras que entre los años 2010 y 2016, los ejes privilegiados para su aparición es un arco norte-este-sur, con dos apariciones en el sector sur, precisamente en el cantón Desamparados, el cual representa uno de los cantones donde habita mayor cantidad de población en la GAM (figura 3). La figura 3 también permite observar que los malls se localizan, con el pasar de los años, hacia los márgenes espaciales de la GAM, teniendo como punto de referencia al cantón San José.

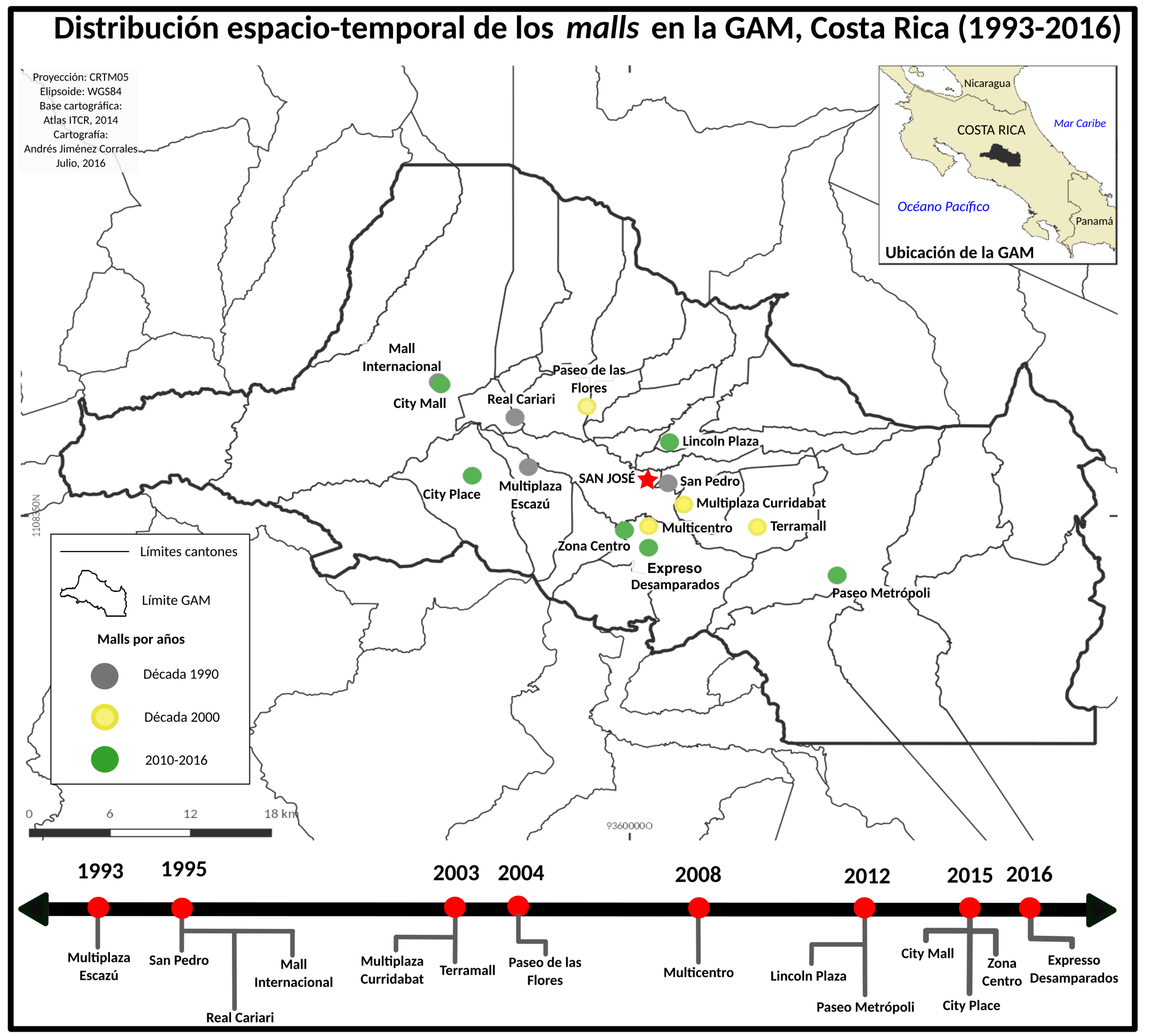


La figura 4 muestra la localización de los malls en la GAM con respecto a la población que habitaba por cantón en el año 2011. Destaca el hecho de que ocho de los 14 malls se emplazan en cantones con una población de más de 81.000 habitantes, lo cual representa más que el promedio poblacional de la GAM en dicho año (65.616 personas). Si bien esta variable está lejos de ser definitoria para la localización de un centro comercial de este tipo debido a que esta decisión responde a una combinación de variables, como puede ser el caso del valor de la tierra, sirve para denotar una característica que muestra el espacio donde se están emplazando los malls, lo cual puede ser utilizada a su favor, hay una población cautiva de consumidores en su espacio contextual.

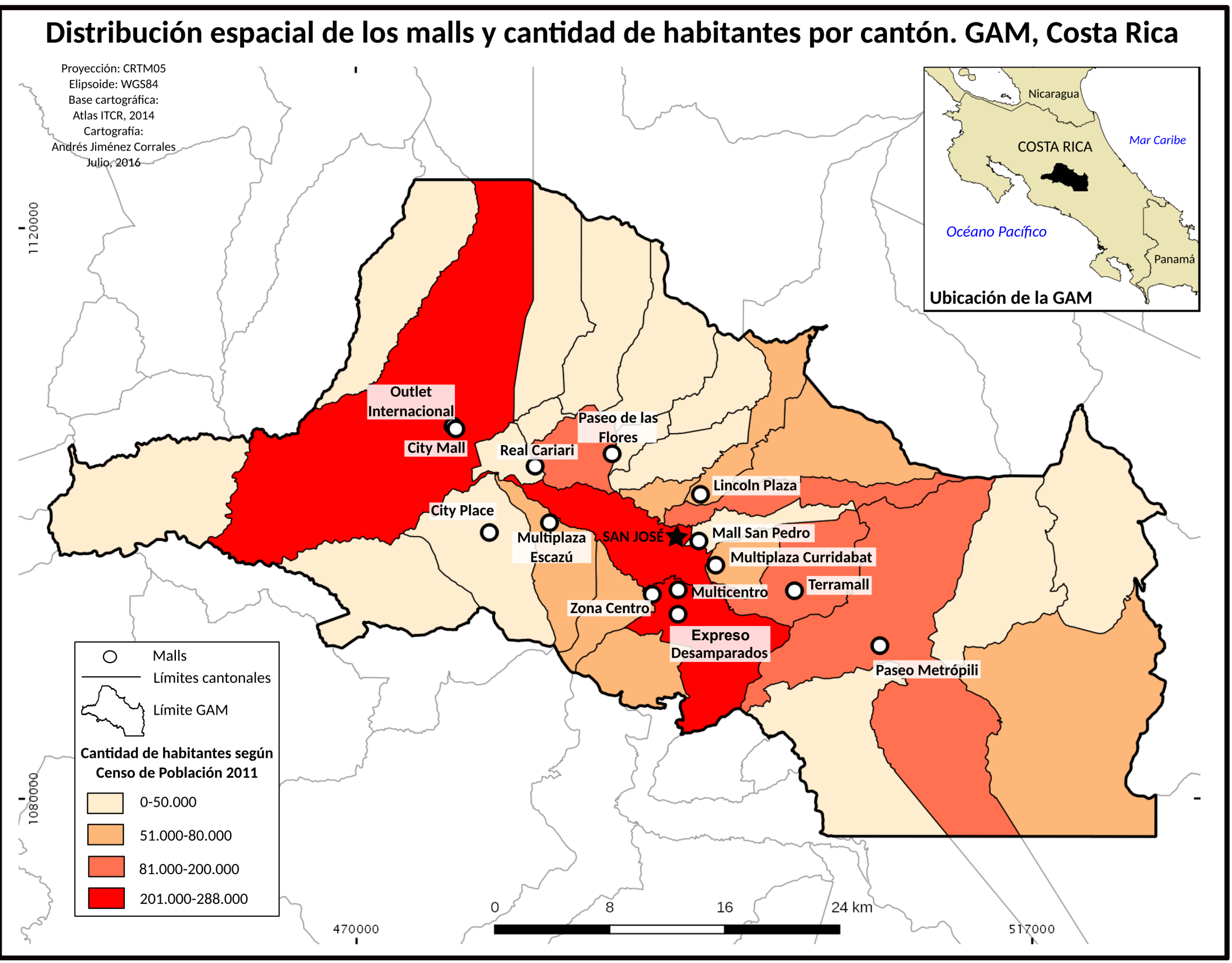

Figura 4. Ubicación de los malls en la GAM (2016) y cantidad de habitantes por cantón (2011). Fuente: INEC, 2011. Elaboración propia
Otro aspecto importante a considerar en la ubicación de los malls es su relación con vías de circulación, tal es el caso de:

1. Multiplaza Escazú frente a la Ruta nacional $n^{\circ} 27$,

2. Terramall y Paseo Metrópoli frente a la Ruta nacional $n^{\circ} 2$ (autopista Florencio Castillo),

3. Mall San Pedro contiguo a la Ruta nacional $n^{\circ} 39$ (Circunvalación),

4. Real Cariari sobre la Ruta nacional $n^{\circ} 1$ (autopista General Cañas),

5. Mall Internacional y City Mall sobre la carretera Radial a Alajuela,

6. Paseo de las Flores sobre Ruta nacional $n^{\circ} 3$ (una de las entradas a la ciudad Heredia).

Esto demuestra que en la entrada de las ciudades Alajuela, Heredia y Cartago, cabeceras provinciales, se han emplazado malls. Esto permite pensar en el proceso de relocalización de centralidades, pasando de un centro administrativo, territorio municipal, a un centro comercial, territorio de un actor empresarial. 
A pesar de que la territorialización de los malls, al año 2017 cuenta con 24 años de desarrollo, en el país ha estado lejos de instaurarse una "industria del mall". Lo que se demuestra en una ausencia de elementos divulgativos como una revista o un grupo que congregue a los inversores, como lo puede ser una asociación o una cámara tal como sucede en otros países como Estados Unidos o, recientemente, en Chile (Salcedo y De Simone, 2012, p. 19). Este hecho permite reflexionar que las condiciones en las que se ha desarrollado la territorialización de los malls, ha estado lejos de crear una necesidad de congregar a los actores empresariales en un grupo que sirva para defender sus intereses o promover los beneficios de su inauguración. Este elemento puede señalar, en parte, el nivel de aceptación social de estos espacios, lo que les da un poder para transformar geografías.

Además se pudo comprobar que en los instrumentos de planificación territorial regional como el Plan GAM 82, en la propuesta PRUGAM y en el Plan GAM 2013, se está lejos de normar la ubicación de los malls. Ante este hecho, el ex director de urbanismo del INVU, Leonel Rosales, señala una consecuencia de no planificar la localización de estos espacios:

“...es una necesidad [planificar] porque hay que ver el impacto, pero de verdad... por ejemplo el mall Desamparados... lo pusieron en el área más congestionada... en el centro de la ciudad, y no resolvieron los problemas de accesibilidad a la ciudad..." (L. Rosales, entrevista, 27 de octubre del 2015).

Este punto se convierte en un vacío aprovechado por los actores empresariales para generar su propia planificación. Ante este hecho, el ex director de urbanismo del INVU es enfático en señalar que la regulación para los malls no se debe de limitar tan solo a establecer en un plan regulador dónde se puede o no se puede instalar un espacio de este tipo. Por lo que se debe pensar en legislar en temas en específico, entre ellos obligar la elaboración de un plan maestro que colabore a integrar una serie de variables dentro del desarrollo (L. Rosales, entrevista, 27 de octubre del 2015).

\section{Reclamos y respuestas ciudadanas ante la territorialización de los malls}

Hasta el momento se ha mostrado el proceso de establecimiento de los malls en la GAM. Para esto se han señalado los cantones y los años de instalación de estas infraestructuras, pero también se han indicado algunas características de su localización. A raíz de esto se quiere completar el análisis denotando algunos de los reclamos y las respuestas ciudadanas realizadas ante la llegada de un mall a un lugar.

A partir de una recopilación de noticias de prensa nacional, realizada para esta investigación, se determinó que uno de los principales reclamos ciudadanos ante el establecimiento de un mall gira alrededor de posibles aumentos de congestión vehicular, preocupación que se encontró también en actores institucionales. Por ejemplo, Maureen Fallas, alcaldesa del cantón Desamparados del periodo 2006-2010, reaccionó de esta manera ante la construcción del mall Multicentro: "Nos preocupa mucho el impacto que el centro comercial pueda tener sobre el tránsito, porque el ingreso al centro de Desamparados ya es complicado" (Delgado, 18 de agosto del 2008). Dicho comentario refleja que el actor institucional asume una reacción pasiva ante este hecho, dejando de lado el papel que le ha sido asignado en la normativa nacional como el actor regente de la planificación territorial local.

Con la construcción del mall American Outlet ${ }^{11}$ se dio un hecho similar, donde algunos vecinos reclamaron que con la entrada en funcionamiento de este centro comercial se iba a generar mayor congestionamiento vial, catalogando la situación previa como un caos. A raíz de esto, la Municipalidad de Montes de Oca sale en defensa del mall, exponiendo una serie de obras que van a realizar para evitar que dicho escenario suceda.

De manera similar, el Ministerio de Obras Públicas y Transporte (MOPT), reacciona ante la denuncia pública señalando la contratación de un estudio para el desarrollo de recomendaciones para mejorar la organización del tránsito en las inmediaciones 
del mall (Loaiza, 22 de septiembre de 1998; y Loaiza, 6 de abril de 1999). Este caso permite determinar la forma como los actores institucionales realizan acciones que se encuentran influenciadas por la actuación de actores empresariales, por lo que buscan adaptar la dinámica contextual al establecimiento de estos espacios.

Otro de los reclamos recurrentes gira en torno a efectos adversos que puede ocasionar el establecimiento de un mall al ambiente. Por ejemplo, la corta de árboles en zonas de protección de ríos o la contaminación de ríos. Esto se pudo comprobar en el caso de la construcción de un mall que se iba a llamar Center Mall (ubicado en Heredia). Para este caso se denunció en la prensa nacional los daños efectuados por el actor empresarial en el área de protección del río Pirro (corta de árboles sin los permisos correspondientes) (Loaiza, 3 de marzo del 2004; Loaiza, 31 de marzo del 2004).

A pesar de lo señalado se logró determinar que los reclamos de los actores locales hacia la instalación de los malls, representan procesos aislados que han carecido de fuerza para crear un movimiento ciudadano que convierta en discusión pública el establecimiento de este tipo de espacios. Esta situación puede estar potenciada por el hecho de que un mall representa un espacio de concentración de bienes y servicios que se introduce de manera fácil en la cotidianidad de las personas, ya que existe un faltante de espacios que reúnan estas características. En este sentido, un vecino del cantón Desamparados expresa, ante el anuncio de la llegada del mall Multicentro, que "el mall es una buena idea para no tener que salir de Desamparados. Ojalá terminen pronto la construcción porque hacen falta los cines" (Delgado, 18 de agosto del 2008). Dicha opinión refleja un reclamo hacia la falta de espacios que brinden una oferta de servicios culturales-recreativos cercanos a su sitio de habitación. Por lo que un actor empresarial aprovecha esta situación para generar un espacio con esas características, lo que permite que las personas confluyan en el espacio que es creado y, con ello, logran constituirse en una centralidad.

Los malls se han convertido en espacios de confluencias de familias, amigos y parejas, por lo que han sido catalogados como "el lugar predilecto para invertir el fin de semana" (Gólcher, 25 de junio del 2000). Ante esto, el sociólogo Jorge Hidalgo (citado por Gólcher 25 de junio del 2000) expresa que los malls han ganado ese lugar de privilegio debido a que en el Área Metropolitana no existen tantos espacios recreativos, por lo que los centros comerciales se convierten en el punto de encuentro más indicado para tales efectos.

Los actores empresariales establecen reglas internas por lo que les permite generar una apropiación y ejercer un dominio en su espacio; por ende, en las personas que visitan estos lugares. A raíz de ello se han dado situaciones donde a algunas personas se les ha negado la entrada al mall. Por ejemplo en el mall San Pedro, en el año de 2012, oficiales de seguridad obligaron a salir del lugar a una pareja homosexual. Los ofendidos denunciaron el hecho ante la Sala IV, cuyo resultado fue ordenar al mall la no discriminación de parejas de orientación sexual diversa (Acuña, 19 de mayo del 2012). Otro ejemplo fue un caso acontecido durante el año de 2013 en el mall Lincoln Plaza, cuando a una mujer que se encontraba amamantando a su hija en el espacio de comidas se le prohibió realizar esta acción en público. Para ello, una oficial de seguridad le indicó a la madre que no podía realizar esta acción ahí debido a que existían salas de lactancia hechas específicamente para ello. Ante la denuncia pública interpuesta por la madre hubo distintas reacciones, entre ellas la de Lincoln Plaza, quienes en su página de Facebook expresaron: "Les dan una sala para que puedan estar más cómodas con sus bebés y se quejan. Cómo es la gente". Minutos después este comentario fue borrado de la red social (Ross, Loaiza y Agüero, 8 de enero del 2013). Estos acontecimientos remiten a pensar en otras poblaciones que no son aceptadas en estos lugares, como es el caso de las personas en situación de calle, que en relatos recabados para esta investigación, se identificaron casos donde se presenció una prohibición a su entrada.

Con lo expuesto se observa que alrededor del establecimiento de un mall existen opiniones contrapuestas. Algunas personas se oponen a las consecuencias que genera su proceso de construcción, mientras otras personas desean la inauguración de estos espacios. Además se denota que un elemento central en la producción territorial de los malls es generar reglas que señalan lo que se permite en su interior, generando límites entre su espacio y el afuera. Lo anterior refleja que existe un control territorial que 
disciplina cuerpos (señala qué se puede realizar y de qué forma) y ordena un espaciotiempo (Haesbaert, 2011, p. 125).

\section{A modo de cierre}

Al año 2017 el proceso de territorialización de los malls en Costa Rica contabiliza 24 años de trayectoria. Distintos actores empresariales han creado estos espacios, ubicando sus desarrollos de manera estratégica en la ciudad, creando una oferta de un espacio que antes del año de 1993 era inexistente.

El sector privado, desde la década de 1990, ha aumentado su participación en la construcción de áreas residenciales, comerciales y recreativas, lo que les ha permitido tener un papel preponderante en el desarrollo urbano de la GAM. Un aspecto que agrava este escenario, y en relación nada más con los malls, es que ningún instrumento de planificación urbana establecido o propuesto, contempla a estos espacios dentro de sus lineamientos. Con esto se determina que la escasa planificación de los malls favorece a los intereses privados de los empresarios, específicamente a las inmobiliarias del sector de la construcción. Lo que permite una mayor amplitud de acción para establecer infraestructura residencial, comercial o de servicios en el espacio.

La territorialización de los malls se ha consolidado como un proceso sostenido en el tiempo. La década del 2000 fue el lapso donde menos malls se inauguraron. Mientras que los años 2010-2016 representa el periodo con mayor dinamismo en la construcción de malls en la GAM. La localización de estos espacios responde al área construida de la GAM, con un predominio en el eje este-oeste y algunas incursiones en cantones al norte y sur, tomando como referencia a la ciudad San José. A la vez, se refleja que con los años se ha dado un tipo de expansión hacia la periferia en la construcción de los malls.

Los malls representa mucho más que simples infraestructuras para la compra y venta de productos, caracterizándose por ser puntos de encuentro y esparcimiento (Baltodano citado por Briceño, 2014, p. 10). Es así que el Perfil del Consumidor ${ }^{12}$ del año 2015 arroja un dato que permite comprobar la importancia que tienen estos territorios en la vida de las personas que viven en la GAM, ya que determina que los centros comerciales tienen un $94 \%$ de visita de las personas que residen en este espacio ${ }^{13}$. El sector de la población que asiste más son jóvenes entre los 18 y 24 años, activos laboralmente y con un mayor nivel socioeconómico (Fallas, 2 de julio del 2015).

Los malls representan un hito del urbanismo neoliberal, ya que lograron convertir un proyecto particular en uno universal (Janoschka e Hidalgo, 2014) que ha permitido proyectar una forma concreta de vivir y experimentar la ciudad. Lo anterior se ha podido realizar porque estos territorios tienen la particularidad de concentrar tres actividades alrededor de las cuales se da la socialización: 1) intercambio comercial, 2) diversión y 3) recreación (Finol, 2005, p. 575). Es así que la actividad social se realiza en espacios privados que están condicionados por los intereses de los actores empresariales que a través de estos espacios controlan, en parte, nuestras formas de habitar la ciudad.

12 El Perfil del Consumidor es un estudio que realiza Unimer, de manera anual, para el periódico El Financiero. Al año 2016 este estudio se ha realizado en cinco ocasiones. El Financiero promulga que el estudio contempla la actitud de consumo de los costarricenses, basado en nueve categorías (Fallas, 25 de septiembre del 2016). 13 Dentro de dicho porcentaje se consideran distintas tipologías de centros comerciales como son malls, plazas comerciales y centros comerciales. No es posible obtener un dato preciso para los malls.
Sin dudas, el auge de los malls se ha debido a un deterioro del espacio público, el cual cumplía tiempo atrás las funciones que se dan ahora en estos espacios privados. Como responsable de esta realidad se encuentran los distintos gobiernos que, desde la década de 1990, han realizado pocas acciones para mejorar la calidad y seguridad de los espacios públicos (Salcedo y De Simone, 2012, p. 16) traspasando esta función a actores privados, lo que puede ser una exteriorización de procesos de neoliberalización en la ciudad. Lo anterior propicia la territorialización de los malls ya que se crea un monumento que se erige como una solución a esa situación, el cual adopta funciones de la vida en ciudad.

En este contexto queda pendiente realizar un estudio que enmarque los cambios estructurales de reconfiguración de la clase media en Costa Rica a partir de la década de 1990, así como cambios culturales en los patrones de consumo. Estos son dos elementos que pueden contribuir a generar una explicación más completa de la territorialización de los malls. 
Por último, es importante considerar que los malls son espacios que se han convertido en centralidades debido a la oferta de bienes y servicios, lo que es reflejado en su nivel de visitación mensual. Representan espacios de confluencia de personas que aprovechan el abandono que ha tenido el espacio público para constituirse en alternativas de espacios para estar y convivir en la ciudad. Esto refleja el poder que tienen las inmobiliarias para hacer su proyecto hegemónico.

\section{Referencias bibliográficas}

Acuña, R. (19 de mayo de 2012). Sala IV ordena al 'mall' San Pedro no discriminar a parejas gays. Periódico La Nación. Recuperado de www.nacion.com/archivo/ Sala-IV-San-Pedro-discriminar_0_1269473168.html

Amaya, C. (2009). Rol de los centros comerciales en la organización espacial de las principales aglomeraciones urbanas de Venezuela. Rev. Geográfica Venezolana, 50(2), 263-286.

Arce, S. (24 de octubre de 2009). Multiplaza Escazú abrirá su quinta etapa en dos semanas. Periódico La Nación. Recuperado de http://wvw.nacion.com/In_ee/2009/ octubre/24/economia2133781.html

Alvarado, J. (2014). Demanda de espacios comerciales no crece al ritmo de la oferta. Rev. Construcción, 182.

Alvarado, A. y Gustavo, J. (2014). Urbanizaciones cerradas en Costa Rica: Transformaciones socio-espaciales en la urbanidad y segregación socioresidencial en el distrito San Rafael de Escazú (1990-2012). Tesis para optar al grado de Licenciatura en Sociología. Universidad de Costa Rica, San José.

Avendaño, I. (1990). Evolución de los vecindarios, el caso del barrio y la "urbanización" en Guadalupe, Goicoechea (Costa Rica), 1880-1988. Tesis para optar al grado de Licenciatura en Geografía. Universidad de Costa Rica, San José.

Brenes, L. (19 de abril de 2004). Acabó la bonanza comercial del oeste, mas... en el este hay fe. Periódico La Nación. Recuperado de www.nacion.com/In_ee/2004/ abril/19/economia1.html

Briceño, C. (2014). Infraestructura: Centros comerciales y oficentros. Altos desafíos. Rev. Construcción, 182.

Botero, L. (1997). Ciudades imaginadas, identidad y poder. Rev. Espiral, 7(8), 113-145.

Centro Comercial Guadalupe. (Sin fecha). Nosotros. Recuperado de http://www. centrocomercialguadalupe.com/

Corrales, G. (01 de mayo de 2016). Mall San Pedro: El titán que asombró la Costa Rica noventera. Periódico La Nación. Recuperado de http://www.nacion.com/ocio/ revista-dominical/MALL-historias_0_1558044197.html

De Simone, L. (2014). La Ciudad del Consumo: Nuevos roles simbólicos y territoriales de los espacios de consumo en la ciudad latinoamericana. Memoria VII ENEC.

Delgado, D. (18 de agosto de 2008). Desamparadeños temen colapso vial por 'mall'. Periódico La Nación. Recuperado de www.nacion.com/.../Desamparadenostemen-colapso-vial-emmall_0_995500503.html

Fallas, C. (07 de setiembre de 2014). Miniciudades siguen tomando fuerza en el sector inmobiliario. Periódico El Financiero. Recuperado de http://www.elfinancierocr. com/negocios/Minicuidades-Grupo_Cuestamoras-Portafolio_InmobiliarioDistrito_4-Avenida_Escazu_0_586741356.html

Fallas, C. (06 de febrero de 2015). Expansión comercial y de bienes raíces retoma dinamismo en Costa Rica. Periódico El Financiero. Recuperado de http:// www.elfinancierocr.com/negocios/inversiones-expansion-tiendas_de_modaautomotriz-supermercados-tiendas_de_conveniencia_0_679132114.html 
Fallas, C. (02 de julio de 2015). Centros comerciales se vuelven menos atractivos para comery comprar. Periódico El Financiero. Recuperado de http://www.elfinancierocr. com/negocios/Centros-comerciales-vuelven-atractivos-comprar_0_766723330. html

Fallas, C. (12 de setiembre de 2016). Ticos visitan menos los centros comerciales. Periódico El Financiero. Recuperado de http://www.elfinancierocr.com/negocios/ Ticos-visitan-veces-centros-comerciales_0_1029497046.html

Fallas, C. (25 de setiembre de 2016). Los millennials impulsan el consumo en Costa Rica. Periódico El Financiero. Recuperado de http://www.elfinancierocr.com/ negocios/millennials-Perfil_del_Consumidor-Nielsen_0_1036096406.html

Finol, J. (2005). Globalización, espacio y ritualización: de la plaza pública al mall. Rev. Espacio abierto, 14(4), 49-58

Grupo Roble. (Sin fecha). Somos Roble. Recuperado de http://www.gruporoble.com/ somosroble.html

Gólcher, R. (25 de junio de 2000). ¡Vamos al mall! Periódico La Nación. Recuperado de www.nacion.com/viva/2000/junio/25/var1.html

Haesbaert, R (2011). El mito de la desterritorialización. Del "fin de los territorios" a la multiterritorialidad. Madrid: Ed. Siglo XXI.

Hidalgo, A (2003). Costa Rica en evolución. Política económica, desarrollo y cambio estructural del sistema socioeconómico costarricense (1980-2002). San José: Editorial de la Universidad de Costa Rica y Servicio de Publicaciones de la Universidad de Huelva.

Janoschka, M. (2002). El nuevo modelo de la ciudad latinoamericana: fragmentación y privatización. Rev. EURE, 28(85), 80-117.

Janoschka, M. y Hidalgo, R. (2014). La ciudad neoliberal: estímulos de reflexión crítica. En: Janoschka, M. y R. Hidalgo (Ed.). La ciudad neoliberal. Gentrificación y exclusión en Santiago de Chile, Buenos Aires, Ciudad de México y Madrid. Santiago: Pontificia Universidad Católica de Chile.

Jiménez, A. (2016). Cartografía de la territorialización del mall Lincoln Plaza. Moravia, Costa Rica. 2005-2016. Tesis para optar al grado de Licenciatura en Geografía, Universidad de Costa Rica, San José.

Loaiza, V. (22 de setiembre de 1998). San Pedro teme más caos vial. Periódico La Nación. Recuperado de http://www.nacion.com/ln_ee/1998/septiembre/22/pais5. html.

Loaiza, V. (06 de abril de 1999). Nuevo mall listo en diciembre. Periódico La Nación. Recuperado de www.nacion.com/ln_ee/1999/abril/06/economia2.html

Loaiza, V. (03 de marzo de 2004). Objetan un nuevo 'mall' en Heredia. Periódico La Nación. Recuperado de www.nacion.com/ln_ee/2004/marzo/03/pais8.html

Loaiza, V. (31 de marzo de 2004). Setena frenó nuevo 'mall' en Heredia. Periódico La Nación. Recuperado de: www.nacion.com/economia/Setena-freno-nuevo-mallHeredia_0_675332679.html

Lungo, M.; Pérez, M. y Piedra, N. (1992). La urbanización en Costa Rica en los 80. En: Portes, A. y Lungo, M. (Coord.). Urbanización en Centroamérica. San José: FLACSO Costa Rica.

Madrigal, R. (17 de agosto de 2015). En seis años, usuarios de ruta 27 han pagado $50 \%$ del precio de la obra. Periódico Crhoy. Recuperado de http://www.crhoy.com/ en-seis-anos-usuarios-de-ruta-27-han-pagado-50-del-precio-de-la-obra/

Pérez, M. (2010). Cuando los indeseados se congregan en el mall: prácticas socioespaciales de adolescentes en un espacio semi-público. Tesis de la Maestría en Desarrollo Urbano, Pontificia Universidad Católica de Chile, Santiago.

Plan GAM. (2013). Capítulos introductorios. Consejo Nacional de Planificación Urbana y Secretaría Plan Nacional de Desarrollo Urbano. San José: Ministerio de Vivienda y Asentamientos Humanos, Instituto Nacional de Vivienda y Urbanismo e Instituto Tecnológico Nacional. 
Plan Regional Urbano del Gran Área Metropolitana (PRUGAM). (2009). Tomo I, II y III. San José: MIVAH, MOPT, MINAE, MIDEPLAN, MINSALUD, INVU, IFAM, CNFL y AyA.

Programa Estado de la Nación. (2015). Vigesimoprimer Estado de la Nación en Desarrollo Humano Sostenible. San José: CONARE.

Renna, H. (2010). ¿Desde dónde repensar la ciudad neoliberal? Rev. Investigación y Políticas, 1.

Rosales, L. (27 de octubre de 2015) Director de Urbanismo, Instituto Nacional de Vivienda y Urbanismo. Comunicación personal.

Ross, A.; Loaiza, V. y Agüero, M. (08 de enero de 2013). Usuaria denuncia prohibición para amamantar en público en Lincoln Plaza. Periódico La Nación. Recuperado de http://www.nacion.com/archivo/Usuaria-prohibicion-amamantar-LincolnPlaza_0_1316268459.html

Rovira, J. (2004). El nuevo estilo nacional de desarrollo de Costa Rica 1984-2003 y el TLC. En: Flores-Estrada, María y Gerardo Hernández (Eds.). TLC con Estados Unidos contribuciones al debate. ¿Debe Costa Rica aprobarlo? San José: Instituto de Investigaciones Sociales, Universidad de Costa Rica.

Salcedo, R. (2003). Lo local, lo global y el mall: la lógica de la exclusión y la interdependencia. Rev. de Geografía Norte Grande, 30(1), 103-115.

Salcedo, R. y De Simone, L. (2012). Los malls en Chile. 30 años. Santiago: Cámara Chilena de Centros Comerciales.

Salcedo, R. y De Simone, L. (2013). Una crítica estática para un espacio en constante renovación: el caso del mall en Chile. Rev. Atenea, 507, 117-132

Sanhueza, R., Poduje, I. y Alexander, G. (2009). Malles en Santiago. De centros comerciales a centros urbanos. Rev. Estudios Públicos, 114, 219-252 